\title{
Memorias del II Seminario Internacional; aportes del conversatorio Debates Pedagógicos Contemporáneos: una mirada a las Pedagogías Emergentes
}
Comité Editorial: Adriana Ferreira, Ariel Milstein, Cristina Maciel y Noelia Campos

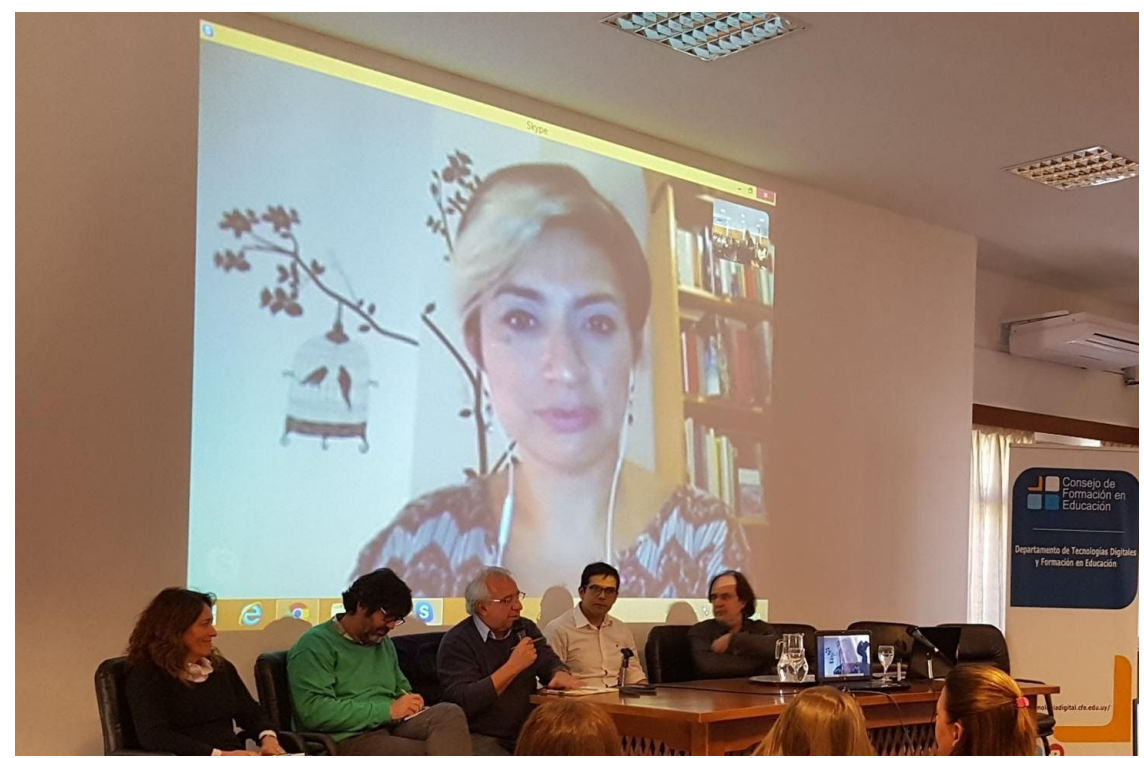

El conversatorio. Foto: Mónica Viera

Moderador: Dr. Enric Prats.

Debate: Dra. Linda Castañeda, Dr. Alejandro Piscitelli, Prof. Gastón Cortés, Dra. Claudia Brovetto y Dr. Antonio Romano.

Esta memoria pretende poner en común los principales hilos argumentales que nutrieron el debate desarrollado en el conversatorio que dio cierre a la 2. ${ }^{a}$ edición del Seminario Internacional titulado La investigación educativa en el contexto tecnológico: Debates Pedagógicos Contemporáneos: una mirada a las Pedagogías Emergentes. Los temas abordados permitieron un interesante vuelo teórico respecto al campo de la tecnología educativa y favorecieron la comprensión del fenómeno de las pedagogías emergentes.

Puede establecerse cuatro ejes sobre los cuales giró el Conversatorio. Estos son:

- La autonomía docente.

- El hackeo a la educación (como forma de conceptualizar las delimitaciones de lo escolar).

- La tecnología y su carácter constitutivo.

- Las pedagogías emergentes. 
Figura 1

Ejes temáticos del debate

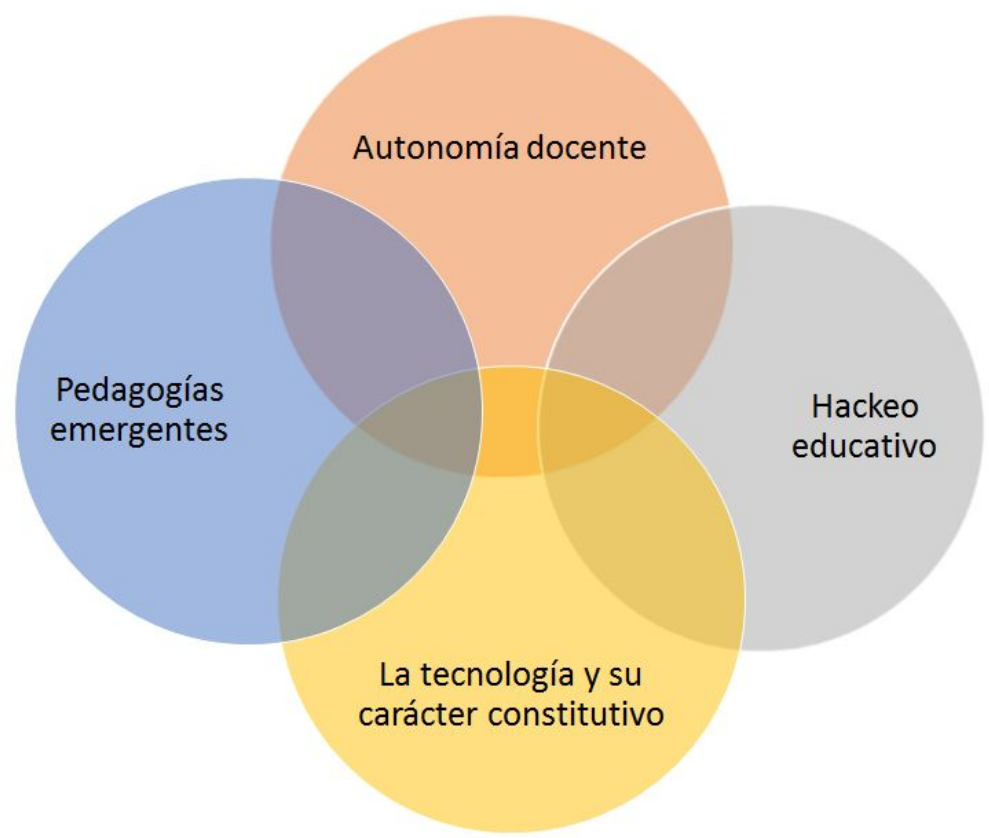

\section{La autonomía docente}

En relación con primer eje, las diversas posturas pusieron en tensión el grado de autonomía que posee el docente en el contexto contemporáneo, no solo frente a sus convicciones y capacidades sino también sobre las condiciones que impone el marco institucional. Al respecto, hubo planteos optimistas y otros que hicieron referencia a una libertad camuflada y a procesos desprofesionalizantes de la figura docente.

Por un lado, desde una mirada optimista, Piscitelli reivindicó el espacio para construir, que es mayor de lo que muchas veces se cree. En este sentido, advierte que no se trata de una mirada ingenua, (a partir de algunas arremetidas del público frente a algunos planteos iniciales). Para él, «en educación hay mucha más autonomía y libertad que en otras esferas laborales donde se tiene varios jefes». No obstante, la distinción entre autonomía real y autonomía aparente estuvo presente en el debate. En ese sentido, Prats coincidió en «distinguir la autonomía virtual de la real». Los obstáculos frente a esa autonomía aparecieron asociados a las presiones que genera el currículo, la jerarquía, el trabajo en organismos burocráticos y la lógica de mercado que atraviesa el mundo exterior y sus cambios.

En suma, ante tantas problemáticas que podrían poner en cuestión la autonomía del docente, aparecieron las advertencias acerca del proceso desprofesionalizador y proletarizador de la figura del docente. Sobre esto, Castañeda planteó que «hay un movimiento claramente desprofesionalizador que empieza en los años ochenta» y que «empezamos a hablar del docente como un operario que tiene que llevar 
adelante una serie de cosas que se las mandan de arriba. $Y$ me parece que deberíamos revertir ese proceso». Complementariamente, y a partir de la intervención inicial de Piscitelli relativa al alto margen de autonomía que hay en educación, una intervención del público añadía: «generalmente, los docentes no lo vemos así y toda la literatura de la proletarización del docente implica la falta de autonomía precisamente para ser un profesional para tomar decisiones».

Finalmente, algunas voces del público señalaron lo problemático de seguir una tendencia educativa actual sin una apropiación crítica: «creo que si nosotros hacemos una experimentación porque estamos convencidos, comprometidos, es una verdadera transformación. Si nosotros hacemos una incorporación de una moda, y bueno... entonces tenemos las computadoras no sabemos bien qué hacer, pero bueno, hagamos algo... Ahí ya en esa transformación yo le pongo unos cuantos signos de interrogación». Castañeda coincidió con esto, afirmando que «por seguir al influencer no tenemos tiempo de pensar en este qué quiere decir ni de leer, reflexionar o apropiarse del proyecto, como decía la compañera. También tenemos margen para hacer, también tenemos cierto margen y ese margen debemos usarlo a nuestro favor, a veces podemos ignorar a un inspector». Romano compartió su preocupación acerca de las implicancias «de estar corriendo detrás de lo que es lo último. Que eso impacta, no hay duda. Que tenemos que pensar cómo incorporarlo, totalmente de acuerdo. Ahora, ¿que tenemos que plegarnos automáticamente a como se configura el mundo para traducirlo en la escuela? Hoy, obviamente, esos espacios de lo que delimitan el afuera del adentro están cambiados. Yo no lo voy a discutir, pero si no hay un espacio que separa, no hay escuela, lo que tenemos es mucho mundo y mucho mercado».

\section{Hackeo educativo}

Un segundo eje que estructuró el debate estuvo vinculado con la metáfora del hackeo a la educación y los espacios escolares. En primer lugar, el aporte de Piscitelli fue central para instalar la idea de que es posible producir alteraciones en la forma escolar. Para ello, se sirvió de un concepto para nada ingenuo: el de hackeo. Hackear una propuesta educativa nos lleva a recuperar un término procedente del campo de la tecnología y la informática. Si bien el hackeo se confunde usualmente con la vulneración maliciosa de la seguridad de programas de software, en realidad el término es mucho más amplio, permitiendo, en muchos casos, detectar fallas en los sistemas para reportarlas a sus programadores, creadores de esos programas. De esta forma, Piscitelli comparte su experiencia en un colegio de México en el cual se desarrolla una propuesta educativa experimental. Así, narra cómo se desarrolló el pentahackeo, es decir, la intervención de cinco dimensiones de la escuela para reinventarlas: el espacio, el tiempo, los contenidos, la evaluación y los vínculos.

De forma complementaria, las voces de Brovetto y Castañeda estuvieron en consonancia con algunos aspectos de las reflexiones de Piscitelli. Para Brovetto, la 
tecnología juega un rol de impulso al cambio dentro de las propuestas educativas. En el marco de la Red Global de Aprendizajes y su desarrollo en nuestro país, sus reflexiones giran alrededor del trabajo por competencias, invitando por ello al público a «pensar cómo se trabajan los contenidos por competencias, porque se trabajan distinto a como se trabajan por asignaturas». Para Castañeda también hay un nudo central en la cuestión de la organización curricular por asignaturas. En ese sentido, afirma que «tenemos el enorme reto de hackear las asignaturas, aunque sigamos trabajando con las mismas. No hay un paraíso al cual retornar, la escuela no es el baladí ahora mismo». Esa organización curricular por asignaturas cristalizadas con el devenir histórico no es, para Piscitelli, un obstáculo a la hora de repensar los saberes que deben ser enseñados en los espacios escolares: "¿Cuáles asignaturas? ¿Por qué las asignaturas que tenemos? ¿Por qué las tenemos divididas? Busquemos otro modelo. Si ustedes se meten en la página del Media Lab del MIT, que es el mejor laboratorio de innovación del mundo, van a encontrar que las investigaciones son todas no interdisciplinarias ni transdisciplinarias, son antidisciplinarias y son indisciplinarias. Esa Biología que nosotros enseñamos en la primaria y en la secundaria es la Biología de hace veinte años. No es lo que está pasando ahora. Entonces no es correr detrás de la novedad, es, a ver..., primero por qué está dividido en disciplinas».

No obstante, Romano pone en cuestión esta idea de que hay que hackear los espacios educativos y las implicancias de esto. Para fundamentar esto, esgrimió una perspectiva distinta a la de los exponentes anteriores. Así, la preocupación de este se vincula con la necesidad de delimitar el adentro y el afuera de lo escolar: «hay mucho mundo y poca escuela. La tecnología ha tomado el mando del mundo, hay que buscar un balance, hacer frente al mercado y la tecnología evoca en parte la presencia del mercado en el aula. No hay que hackear todo lo que sucede en la escuela».

\section{La tecnología y su carácter constitutivo}

Relacionado con los dos ejes ya presentados, aparece un tercer hilo argumental a partir del cual se desarrollan interesantes reflexiones acerca del carácter no neutro de la tecnología. Esta idea de la tecnología como constitutiva aparece, por una parte, de la mano de Castañeda, quien afirma que «no solo depende del uso que hacemos de la tecnología; la tecnología no viene meramente cargada de fábrica, ¿o creéis que los que hacen eso lo hacen neutral, de que cada uno haga lo que quiera? No, no es así, si fuera así la cosa no sería tan grave». Para Romano, esta no neutralidad no se suscribe únicamente a la interacción con el artefacto en sí, sino también en relación con todo lo que gira alrededor de los dispositivos tecnológicos. Tal como hemos visto en sus afirmaciones precedentes, en tanto que «la tecnología ha tomado el mando del mundo" y el desdibujamiento de las fronteras de lo escolar hace que encontremos una fuerte filtración del mercado en el aula a partir de la presencia de la tecnología. Frente a esto, llama a la búsqueda de un balance que recupere esa delimitación de lo escolar. 
Para Piscitelli, las posiciones nostálgicas pueden derivar en cierta ingenuidad, dado que es pensar en un pasado que no va a volver a ser. En esta línea, respondiendo a Romano, añade que si «el software tomó el mando es porque, justamente, lo que estamos aceptando es que la tecnología no es instrumental, la tecnología es constitutiva [...] Google ¿sabés cómo se presenta desde hace años? Como la base de datos de las intenciones de la humanidad. Si nosotros no entendemos que somos hablados por la tecnología, si no entendemos que somos manipulados y nosotros dimos un consentimiento tácito y pasivo por los algoritmos, estamos en grandes problemas. Entonces, lo que tenemos que hacer es devolverles a ellos la densidad histórica, la riqueza de la complejidad, la capacidad analítica y, sobre todo, una cosa que jamás te va a dar una computadora que es el poliperspectivismo, o sea poder ver algo desde muchos puntos».

\section{Pedagogías emergentes}

Por último, se realizó un intercambio acerca del fenómeno de las pedagogías emergentes, concepto que, precisamente, enmarca y nombra esta segunda edición del Seminario Internacional. En primer lugar, vale señalar lo problemático de hablar de pedagogías emergentes, en tanto se trata de un concepto polisémico, poco teorizado aún, y con pocos acuerdos al respecto. Es por ello que Castañeda afirma que «pedagogía emergente se ha transformado en un término bandera de conveniencia». En tal sentido, introduce su reflexión a partir de algunas interrogantes: sobre pedagogía emergente, "¿estamos hablando de aceite de serpiente de ese que lo cura todo? Se dicen muchas cosas y a veces no tenemos muy claro qué se quiere decir. Sobre todo, qué es lo que emerge de las pedagogías emergentes. En cuanto hacemos una pedagogía emergente ¿se nos arregla la clase? ¿los alumnos aprenden más? ¿nosotros somos más guapos?». A continuación de esta intervención, aparecen algunas preocupaciones en el público en las que se denuncia cierto vacío de teorización, aunque, principalmente, en relación con los programas sobre tecnología educativa.

Algunos de los expositores uruguayos aludieron a algunas propuestas emergentes específicas, tales como las Aulas Invertida o Aprendizaje Basado en Proyectos. Estos entienden que este tipo de propuestas pedagógicas presentan rasgos distintivos respecto de los métodos más tradicionales de la enseñanza. Respecto al Aula Invertida, Cortés enfatiza en que el «concepto de aula invertida remite a la posibilidad de invertir los tiempos de gestión del aula». Por su parte, Brovetto advierte que los contenidos se trabajan de un modo distinto en una enseñanza basada en proyectos o con foco en las competencias. No obstante, Piscitelli pone en cuestión que los saberes precisos o técnicos sean desplazados por metodologías flexibles, como, por ejemplo, con el Edupunk. De esa forma, ironiza lanzando su pregunta al público: «¿quién se subiría a un avión donde el piloto se formó a través de una metodología Edupunk? ¿O quién se operaría con un médico o se haría una casa con un arquitecto que haya tenido este tipo de formaciones?». 
Asimismo, Piscitelli toma distancia de cualquiera de las pedagogías emergentes, dejando entrever que no se trata de embanderarse con ninguna de ellas, sino con otras cuestiones de fondo. Así, sentenció categóricamente estas cuestiones en el conversatorio: «Jactarse de ser un docente que trabaja en Aula Invertida, o en PLE o gamificación es errarle, porque eso rápidamente caduca. Lo importante es estar siempre en movimiento. Creemos que estamos haciendo una revolución educativa y en realidad de vuelta... la escuela reformó la reforma. Entonces no por el afán de la novedad, sino para darnos cuenta de que no vayamos a pensar que ninguna de estas metodologías y tecnologías va a resolver los problemas de la transmisión, los problemas de la transferencia y los problemas que son fundamentales».

\section{Otros aportes destacados}

Por otra parte, los panelistas realizaron otros aportes, no enmarcados específicamente en ninguno de los ejes antes comentados, pero valiosos para la reflexión. Compartimos a continuación una selección de ellos:

1. «La educación tiene que servir para entender la complejidad, y la complejidad no se entiende escuchando a docentes en la clase hablando dos horas y anotando lo que dicen. Necesitamos que cada aula sea un laboratorio de innovación que logre hacer converger las ciencias y las artes». (Piscitelli)

2. «En este mundo de influencers y youtubers, también hay influencers educativos». (Castañeda)

3. «El libro de texto, aunque esté en crisis, y lo sabemos, ha cumplido funciones importantísimas que la tecnología debería plantearse. Por ejemplo, el libro de texto sirve como hilo conductor para la docencia, le marca al docente lo que tiene que hacer. El libro de texto le marca una lógica epistemológica y didáctica, le marca también cuál es la fuente de autoridad... Lo dice el libro». (Prats)

4. «No sé qué quiere decir enseñar cosas nuevas en la educación. Es más, creo que no es posible pensar el cambio si no es en un marco de una tradición. Si no, lo que hay es el arrasamiento, o estar inventando la rueda todo el tiempo de vuelta. Yo creo que no hay forma de pensar si no es a través de una tradición». (Romano)

\section{Síntesis}

En suma, podemos afirmar que se trató de un conversatorio en el que se desarrollaron sendos debates sobre las pedagogías emergentes en el contexto contemporáneo. Esto permitió una conversación que conjugó diversos campos de conocimiento (historia de la educación, innovación educativa. tecnología educativa, filosofía de la educación) y desde diversos contextos geográficos (España, Argentina y Uruguay). La complejidad del tema desborda cualquier conversatorio que se pueda realizar, pero, aun así, las líneas dialógicas que se abordaron permitieron reflexionar sobre el sistema educativo y las prácticas cotidianas, abordando la autonomía del docente, el hackeo educativo, el carácter constitutivo de la tecnología y las pedagogías emergentes. 
Aportes del conversatorio «Debates Pedagógicos Contemporáneos: una mirada...

En estos debates, la profundidad, la rigurosidad y la reflexión crítica se combinaron con lo satírico y humorístico, planteando así un espacio ameno para el enriquecimiento de todos los asistentes allí presentes. Es por ello que resaltamos el carácter pasional de la conversación en la que el panel de invitados pudo generar sinergia con un público en el que hubo mayoría de docentes que intercambiaron desde lo experiencial y reflexivo de su rol, siendo este un ingrediente fundamental de anclaje de las diversas miradas que se interconectaron.

Los invitamos a acceder al trailer del conversatorio para poder ver la selección del debate que hemos comentado. En la descripción del video, podrán acceder a una versión más ampliada del conversatorio. 